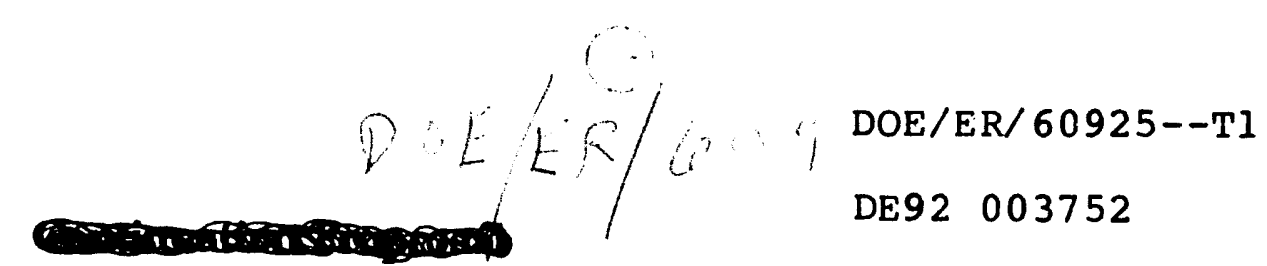

\title{
Skeletal Remodeling Dynamics: \\ New Approaches with Imaging Instrumentation
}

\section{N.J. Parks, K.E. Pinkerton, J.A. Seibert, R.R. Pool, Schools of Veterinary Medicine and Medicine, Unuversity of California, Davis}

This report of progress and future objectives timetable is based on the schematic of goals and objectives on the following page and the project abstract which is included as Appendix I. Five matters are summarized in the order of (a) novel methods of calcified bone confocal microscopy and reconstruction image analysis of decalcified beagle and human cortical bone serial sections, (b) macroscopic cross-correlation of beagle and human cortical and cancellous bone fractions with CT analysis, (c) guidance to the most radiobiologically important skeletal regions of interest with the just completed ${ }^{90} \mathrm{Sr}$ bone tumor map from life time beagle studies, (d) deposition patterns of radioactive agents that participate in apatite crystal nucleation processes in bone and leave radiation-excited electrons trapped in bone mineral, and (e) the budget period timetable. The discovery that beta particles from ${ }^{166} \mathrm{Ho}\left(\mathrm{T}_{1 / 2}=26 \mathrm{hr}, \beta_{\max }=1.8\right.$ $\mathrm{MeV}$ ) phosphonic acid bone agents leave detectable, long-lived, electron paramagnetic resonance signals in bone is included in Appendix II as a joint report (Nature, 24 Jan. 1991, p. 287) with NIST (Radiation Interactions).

\section{(a) Examination of thick-section confocal microscopy as a tool for the} histomorphometric analysis of bone. The advantage of laser confocal microscopy with respect to conventional light microscopy is that it can focus on a very small depth of field (ca. $0.3 \mu \mathrm{m}$ ) by not allowing any out-of-focus light to reach the photodetectors. The depth of field for a particular confocal system can be controlled with the effective aperture. We have used confocal microscopy to acquire the first 3 dimensional images of fluorescent compound deposition patterns in regions of mineralizing bone that simulate the deposition patterns of radioactive materials ( $90 \mathrm{Sr}$ for example) which also deposit predominantly in mineralizing bone. The confocal system allows us to "look ahead" in the range of $50-$ to $100 \mu \mathrm{m}$ into a bone section before it is physically milled away, thus permitting near perfect alignment. Characterization of the 3-D microscopic geometry for these surrogates of radionuclide deposition will simplify and improve the way that radiation doses or toxic agent dosage delivered to the skeleton may be modeled at the cellular level (Parks et al. Joint Bone Radiobiology Workshop, Toronto, July 12-13, 1991, USDOE Report UCD-472-136). We have addressed the issue of how to efficaciously deal with both the biological issues and the technical difficulties that require acquisition and storage of multi-megabyte quantities of data.

We have used a BioRad@ MRC 600 confocal system to study beagle femur and horse cannon (third metacarpal) bone sections from animals labeled in vivo with fluorescent bone labels. The bone sections were obtained, embedded in plastic and cut into nominal $1 \mathrm{~cm}$ sections. Their fluorescent labels were illuminated with the argon laser at $514 \mathrm{~nm}$. Images were acquired at the bone specimen surface and then down variable depths to $50-60 \mu \mathrm{m}$ into the specimen by confocal microscopy. Sections of the bone were then milled off in micrometer increments with a Reichert-Jung milling machine, and confocal microscopic examination was repeated on the subsequent $50-60 \mu \mathrm{m}$ of bone. The confocal images of fluorescing haversian systems were then compiled into a 3-D images.

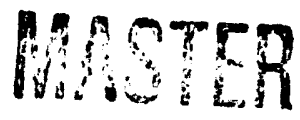




\section{DISCLAIMER}

This report was prepared as an account of work sponsored by an agency of the United States Government. Neither the United States Government nor any agency thereof, nor any of their employees, makes any warranty, express or implied, or assumes any legal liability or responsibility for the accuracy, completeness. or usefulness of any information, apparatus, product, or process disclosed, or represents that its use would not infringe privately owned rights. Reference herein to any specific commercial product, process, or service by trade name, trademark, manufacturer, or otherwise does not necessarily constitute or imply its endorsement, recommendation, or favoring by the United States Government or any agency thereof. The views and opinions of authors expressed herein do not necessarily state or reflect those of the United States Government or any agency thereof. 


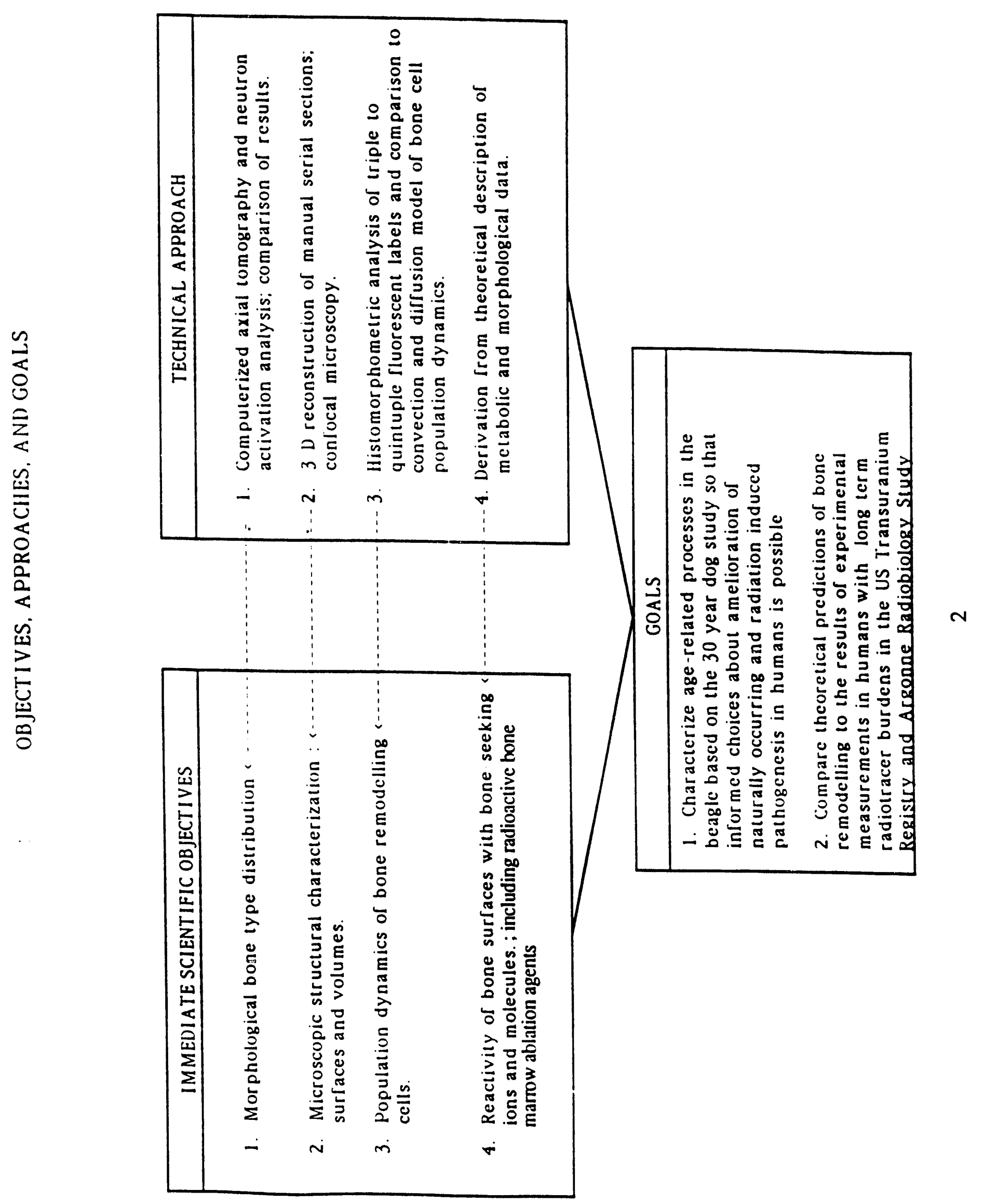


We have evaluated the depths in a bone sample at which resolution is lost by quantifying depths where two concentric labels could no longer be distinguished. This was done with the "column average plot" feature of Image (available from NIH for Macintosh CPU's), which plots the brightness along a selected area. This function is able to display multiple ring profiles that are not clearly defined on the screen (Figure 1).

The depths for which resolution falls within acceptable criteria limits can be measured. The "column average plot" feature (Image) showed some labeled rings were just as bright when scanning $50 \mu \mathrm{m}$ into the sample as they were at the surface. Others deteriorated only 20 $\mu \mathrm{m}$ into the sample. Whether this is the result of true variation in the amount of label present or intervening opacity is currently determined after milling down to the new level.

By combining the use of the confocal microscope and a Reichert-Jung milling machine, we were able to image through hundreds of micrometers of bone in a much shorter time than was previously required for standard morphometric techniques and image acquisition. Once milled, the bone was placed on the confocal microscope where we imaged from the surface down through levels as far as $50 \mu \mathrm{m}$ into the bone. The bone was then milled to a deeper level for further examination by confocal microscopy. Shown in Figure 2 is a false color rendition of a surface image $300 \mu \mathrm{m}$ down from plane zero. The false color rendition expands the gray scale and reveals non-fluorescing structures for orientation.

Serial examination of entire osteons shown required 21 milling operations on bone specimens from the horse and 19 milling operations on the beagle bone. All confocal images were processed using Image (NIH). First, the images are converted to a tagged-image file format (TIFF) for import into Image. Then, by adding the confocal images together, the osteons were aligned using the preceding confocal image to match the selected fluorescent image labels in subsequent sections. Finally, once all the confocal images were aligned, they were compiled into a 3-D image block using Spyglass Dicer® (Figures 3-5). This composite allowed us to examine each entire bone specimen.

The features we wish to illustrate are present in the equine specimens which have been labeled sequentially with combinations of oxytetracycline, calcein green and xylenol orange at weekly intervals, then a 105 day interval, then a similar series of fluorescent labels was given again at weekly intervals, or in beagle femoral cortex with similar labels spaced 14 days apart (Figure 7). The three short spaced labels merged at our resolution on confocal images at the wavelengths we were using. The two merged labels that were clearly defined at $10 \mathrm{X}$ magnification (Figures 1-6) did allow morphometric analysis of bone formed during the 105 day interval.

Two closely aligned osteons were followed in the horse cannon bone. Threedimensional images revealed variation of 50 to $100 \mu \mathrm{m}$ in the diameter of the smaller of the two osteons. The length of bone specimen, i.e., distance of the image series in Figure 6 is approximately $600 \mu \mathrm{m}$. We expect to have the capability conveniently available for sampling accretion patterns directly in 3 dimensions so that the local delivery of radiation flux from bone-seeking radioactive agents to skeletal tissue can be quantified accurately at the microscopic level.

Another important feature is that the orientation and number of the labeled structures is related to local stress and strain in the bone and therefore to remodelling rates and, putatively, to mitotic index in given radiation fields in the case of radioactive bone. In Figure 7, a mineralizing osteon in the proximal beagle femur joins a much larger diameter mineralizing cavity near the endosteal surface and adjacent to marrow spaces. The large structure shows two labels spaced two weeks apart. If formed during ${ }^{90 \mathrm{Sr}}$ feeding of beagles, 


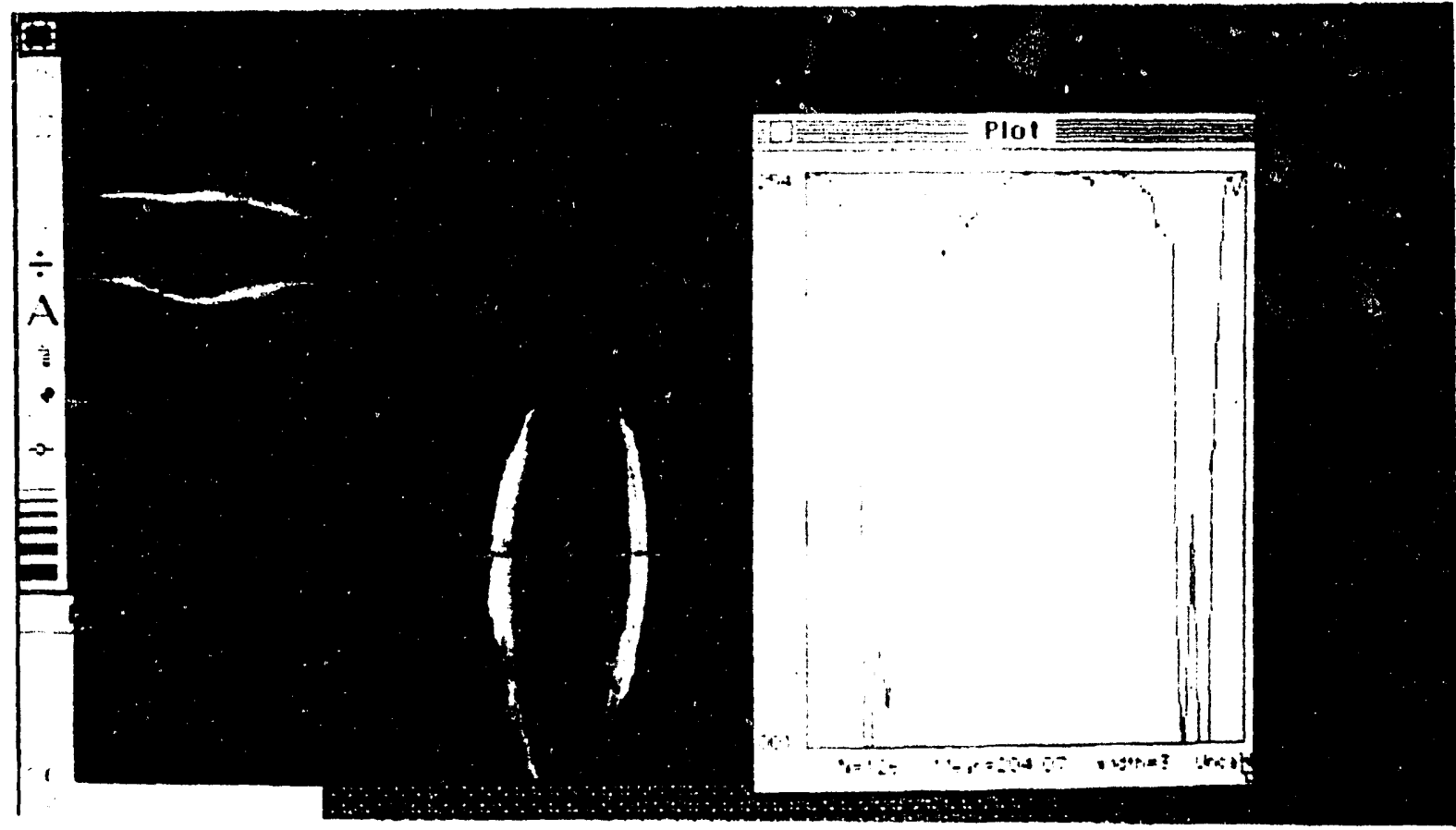

Figure 1. Milled surface of equine metacarpal cortical bone at a plane down $300 \mu \mathrm{m}$ from plane zero. Gray scale display with right structure rotated out from behind plot. Graph displays fluorescent intensity versus distance.

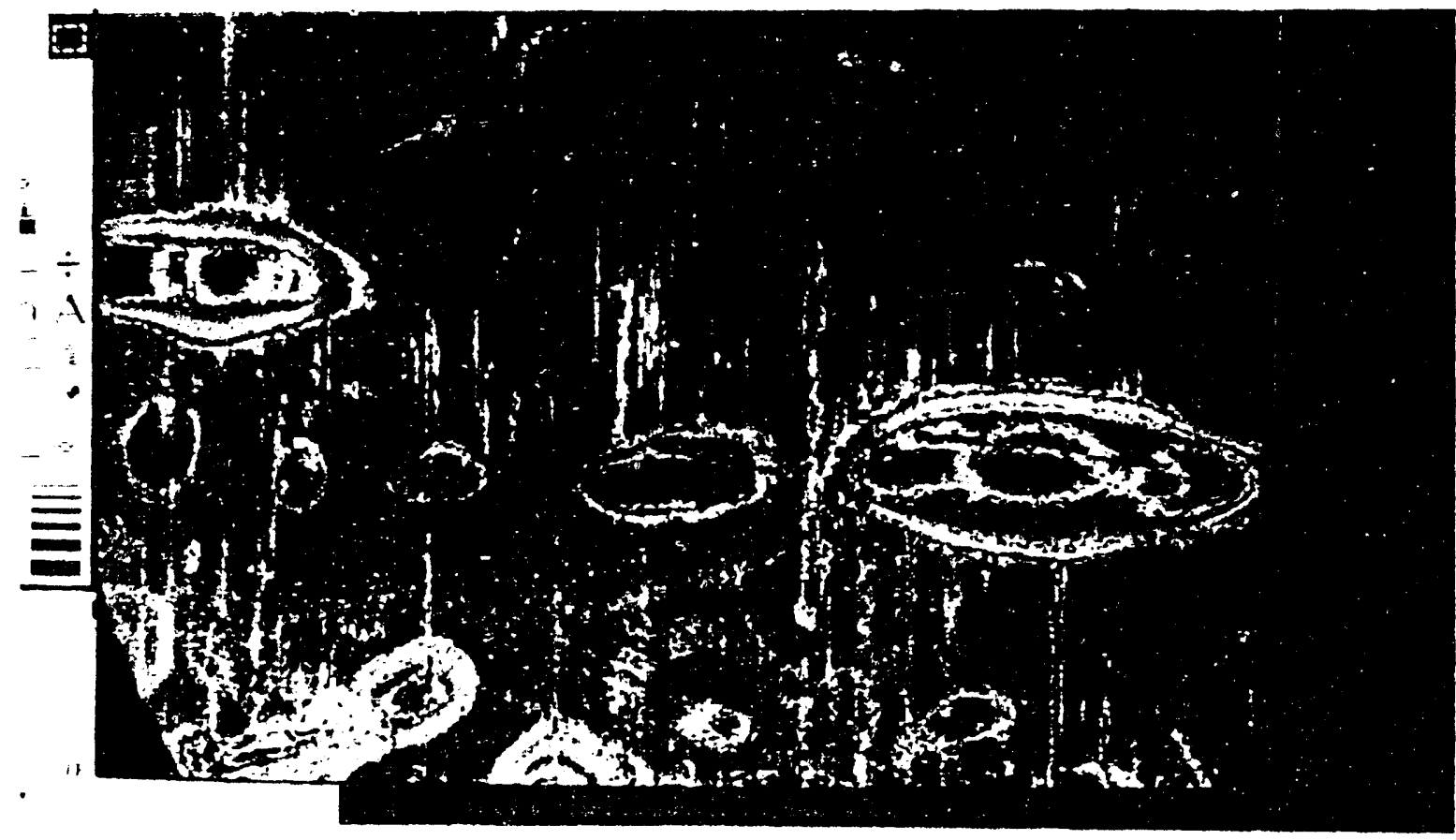

Figure 2. Enhanced image of the same surface as above in its original configuration shows mill marks and other features. Labeled haversian systems are highlighted by false color. Enhancement of original gray reveals non-fluorescing structures which can be followed through the bone. 


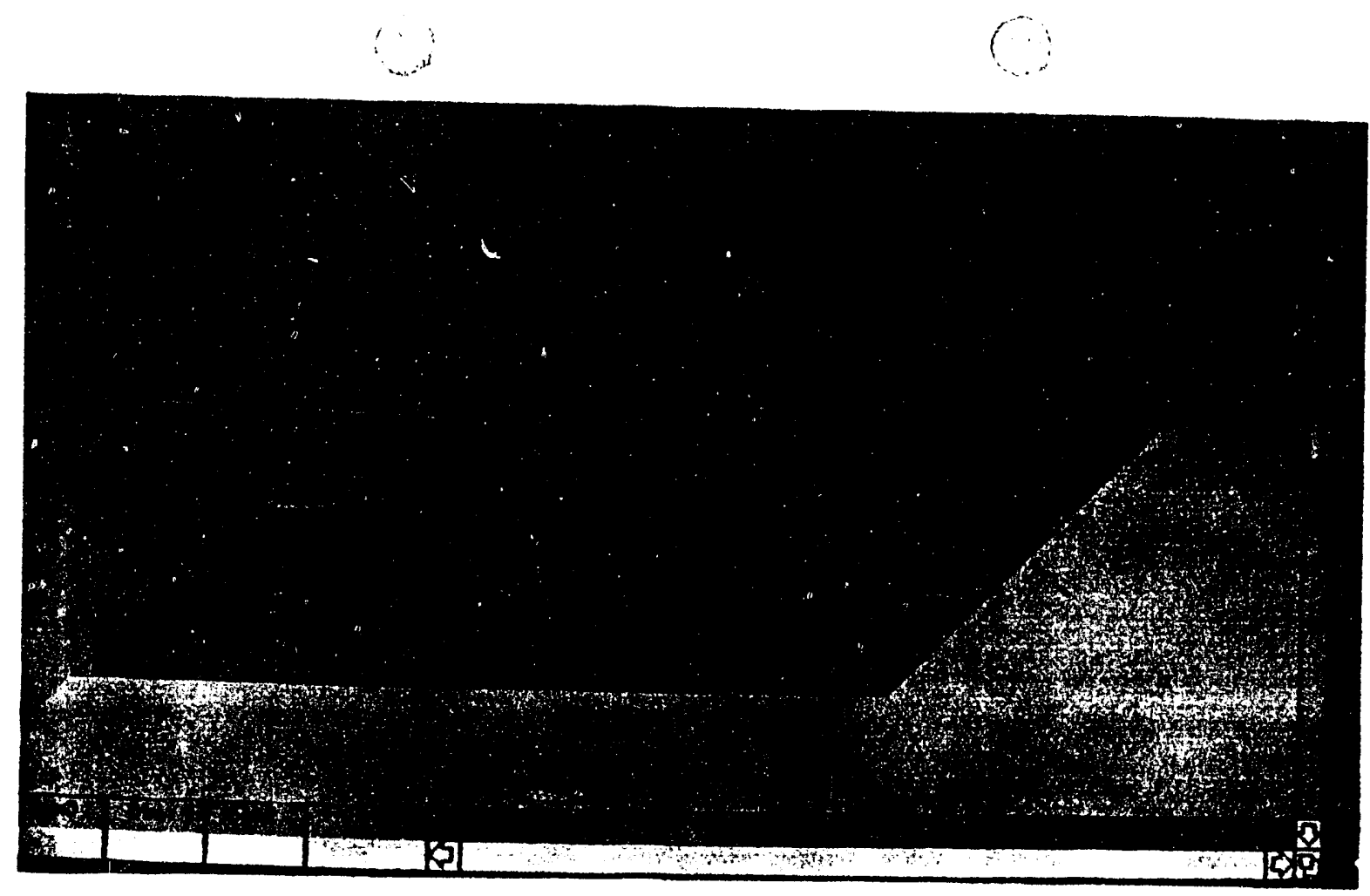

Figure 3. Milled surface of equine metacarpal cortical bone at a plane down $600 \mu \mathrm{m}$ from plane zero.

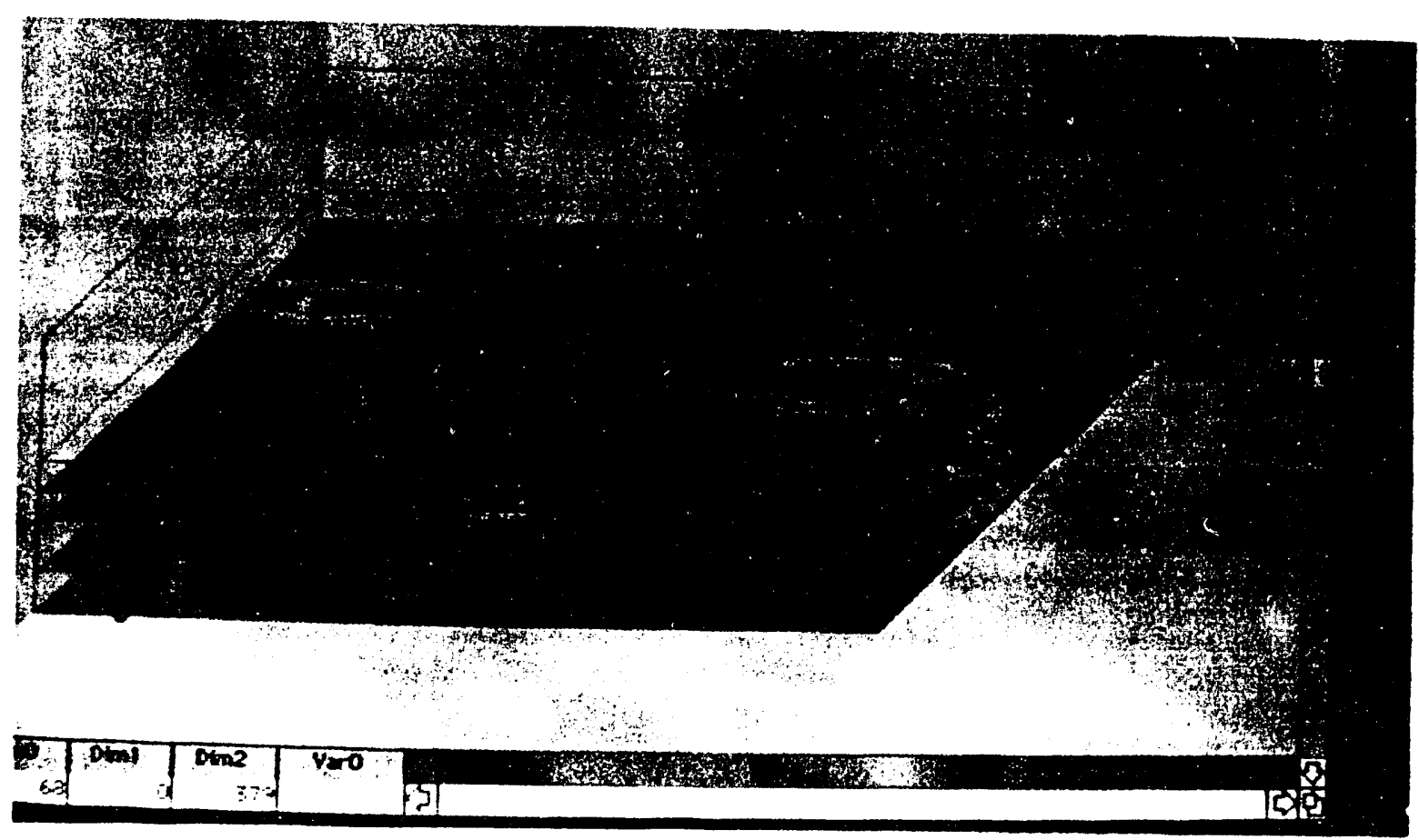

Figure 4. Milled surface of equine metacarpal cortical bone at a plane down $300 \mu \mathrm{m}$ from plane zero. 


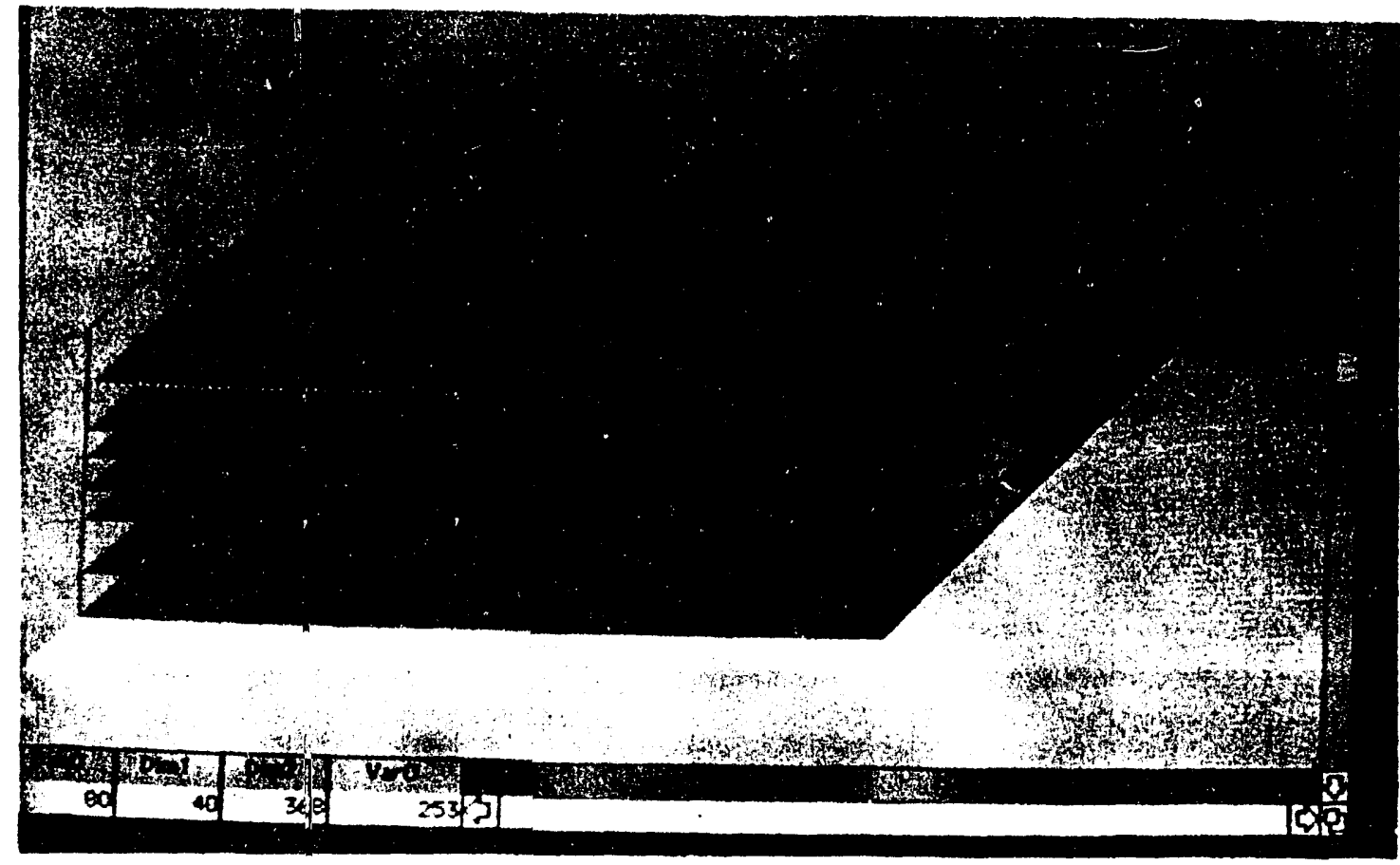

ure 5. Planes at $500 \mu \mathrm{m}$ throica. $100 \mu \mathrm{m}$ showing both fluorescing and nonrrescing structures in the sec.

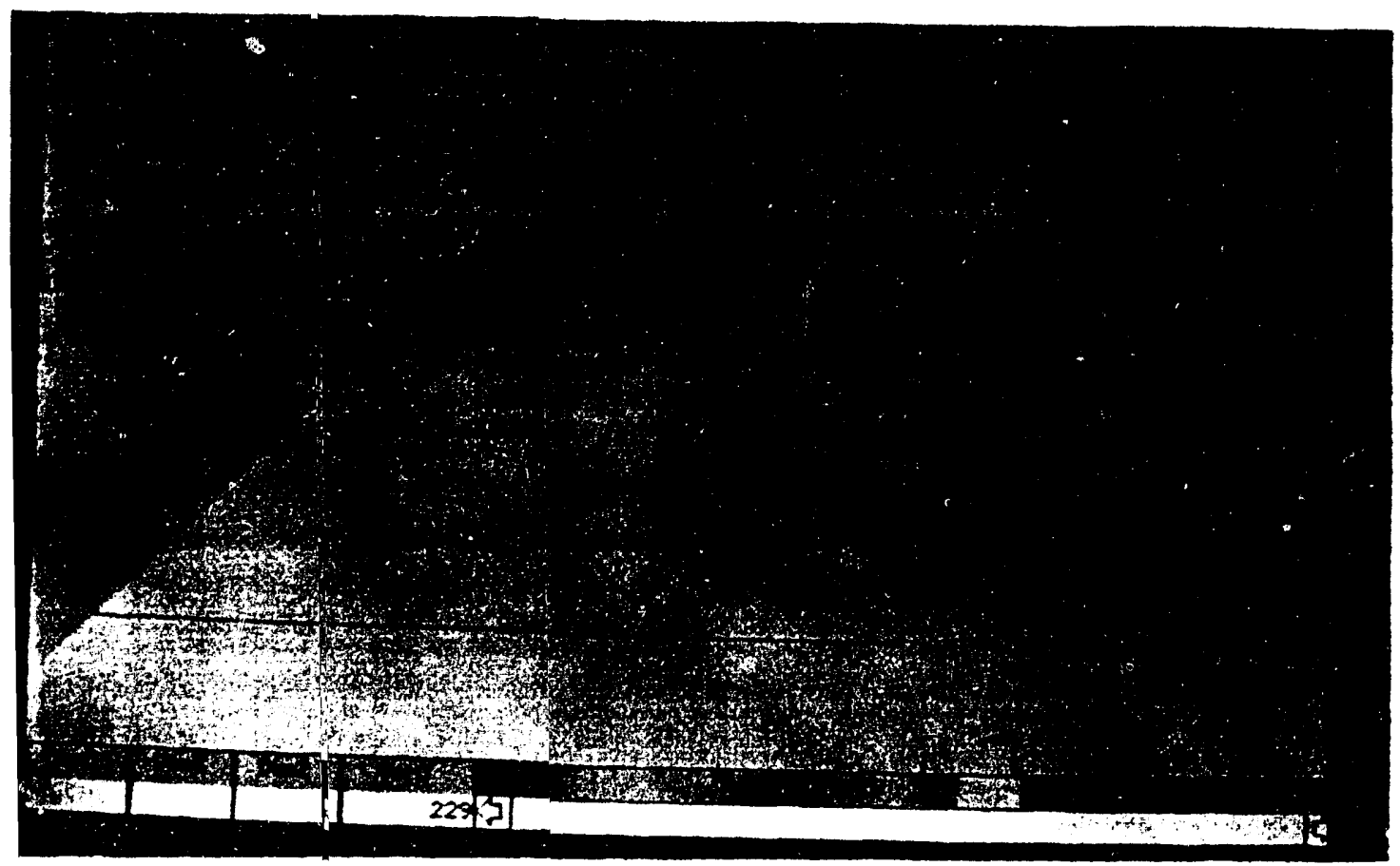

ire 6. Suppressicn of backgrd color for transparency viewing of $600 \mu \mathrm{m}$ of e image planes approximatel $\mu \mathrm{m}$ apart. 


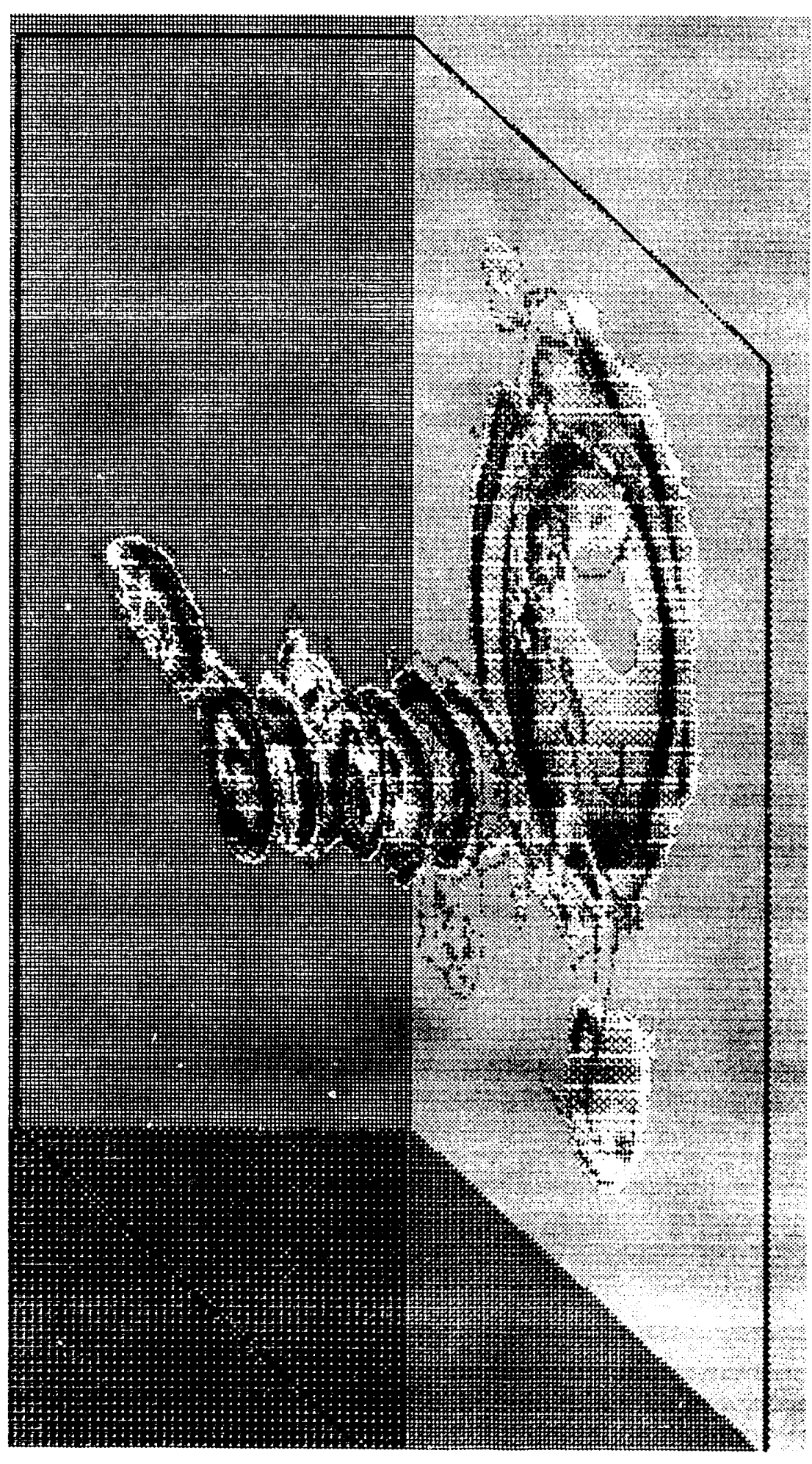

节此

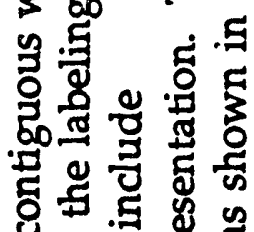
(20.0

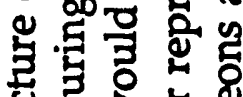
든

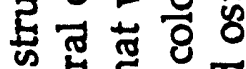
\& of

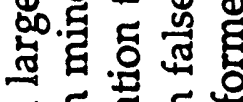
程

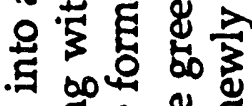
60.5 대욜 可论

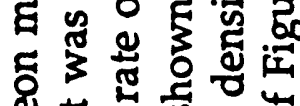
政 (

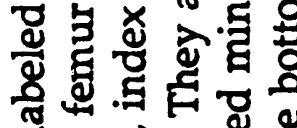

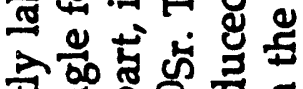
氧。

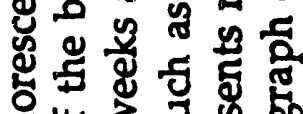

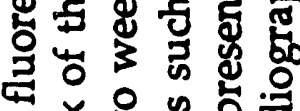

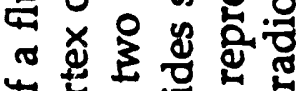
형

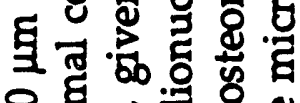
8.0

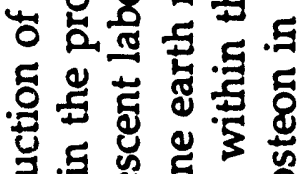

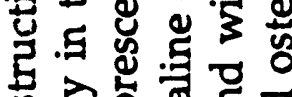

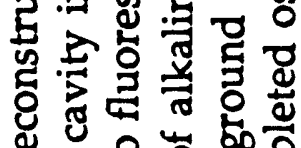
व

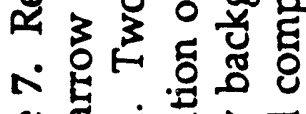

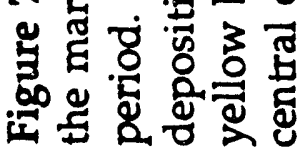


this cortical bone structure would be more radioactive than surrounding areas and is in a region adjacent to marrow stem cell populations. This is important in light of evidence (see ref. Pool et al., section c) that most bone tumors from alkaline earth radionuclides may arise in cortical bone and new evidence that these tumor distributions are random with respect to simple hypothesized relationships to effective surface and bone-type mass (see ref. White et al., section c).

In Figure 8, we show matched images of a confocal micrograph on top and the corresponding microradiograph of a $150 \mu \mathrm{m}$-thick section of cortical bone, displayed for orientation analysis with a 3-D reconstruction program (Spyglass Dicer@) or length and area analysis software (Image, NIH). This demonstrates the ability to connect conventional microradiographic and histomorphometric techniques with what we are doing. Using "look ahead" confocal technique on the thick section before grinding to $30 \mu \mathrm{m}$ or so for conventional fluorescent color micrography, little depth information is lost and all the contextual orientation and microdensity information is gained and can be integrated into the same image files as confocal data. The mechanical, optical, electronic, and data acquisition components of the confocal system are shown in Figure 9.

Figure 10 shows an 18 section segment of decalcified human femur diaphysis stained with osteon darkening silver nitrate (kindly provided by Prof. N. C. Tappen, Anthropology Department, Univ. of Wisconsin, Milwaukee). The sections are $60 \mu \mathrm{m}$ apart. In the ca. 1000 $\mu \mathrm{m}$ shown, there are three mature osteons that we followed for orientation and two that initiate and form around existing mineralized structures that could be radioactive under some conditions of continuous intake. Analogous material for beagle femur sections extends over about $3000 \mu \mathrm{m}$, a range on the order of two osteon lengths. The osteonal orientation in this region, subject to intermittent compression stress induced remodelling, is clearly along the axis of the midshaft.

(b) Macroscopic cross-correlation of beagle and human cortical and cancellous bone fractions with CT analysis. Macroporosities appear with age or the influence of radiation and affect bone density. There are age- and species-related differences in the amount of cancellous and compact bone. Part of the progress we report is that the surprisingly refractory problem of getting the CT number frequency histograms off the primary device and into personal workstations for off line analysis has been overcome. Some salient results of our investigation of CT number density analysis as a way around more tedious physical dissection and neutron activation analysis for quantification of bone type are shown in Figure 11 a,b,c,d,e. We find the integration over entire image regions of interest (ROI) that include all marrow, trabecular and cortical bone gives CT histograms in which the nominal CT number regions of interest (ROI) corresponding to marrow, trabecular bone, and cortical bone readily shows the correlation of similar sections of beagle and human femur. The human data is for a 78 year old male USTR case. Beagle 5750 is 125 months of age and beagle $87 \mathrm{~L} 23 \mathrm{C}$ is 15 months. The shift of some cortical bone to lower CT number nominally characteristic of trabecular bone is apparent in the data of the 125 month old animal. We attribute this to the increase of agerelated macroporosity in the beagle skeleton. It is also clear from our comparisons of relative amounts of cancellous and compact bone as determined by NAA and dissection that quantitative CT gross morphometry must include electronic dissection of the CT images so that cortical bone and trabecular bone ROI densities are separately calculable by comparison to known standards included in the CT field (Figure 11d). 


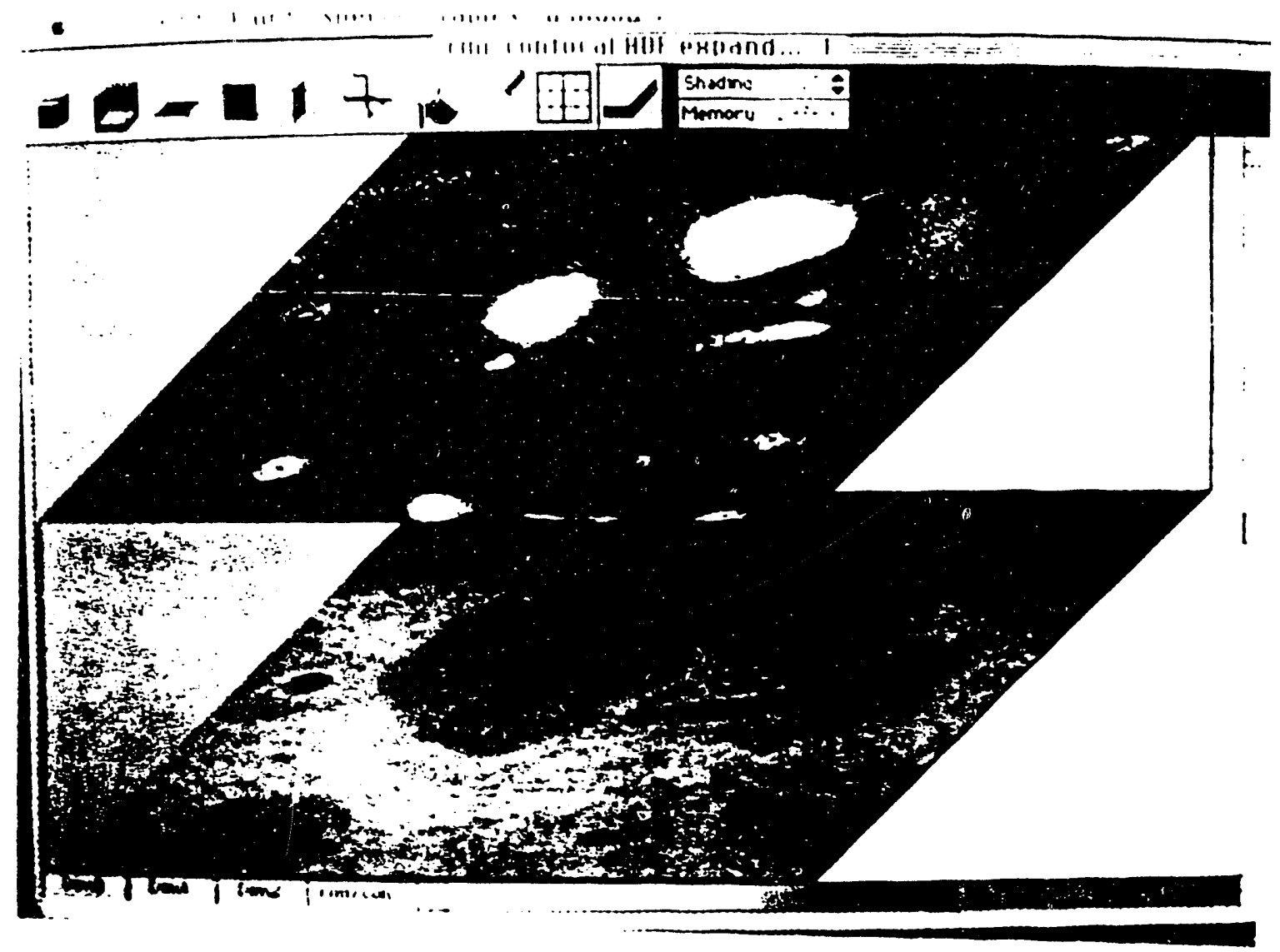

Figure 8. Matched images of a confocal micrograph on top and the corresponding microradiograph of a $150 \mu \mathrm{m}$-thick section of cortical bone, displayed for orientation analysis with a 3-D reconstruction program (Spyglass Dicer) or length and area analysis software (Image, NIH). 


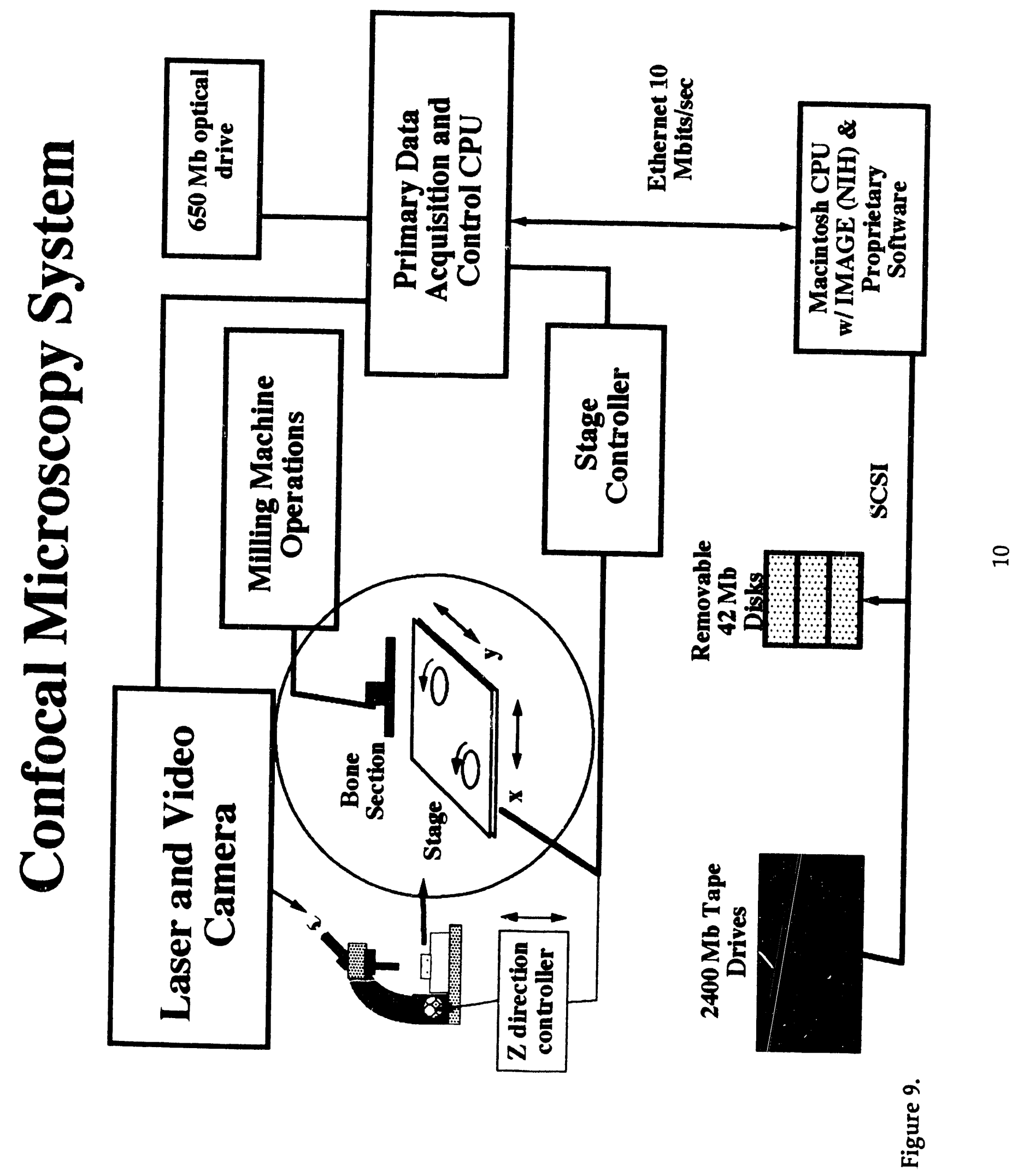




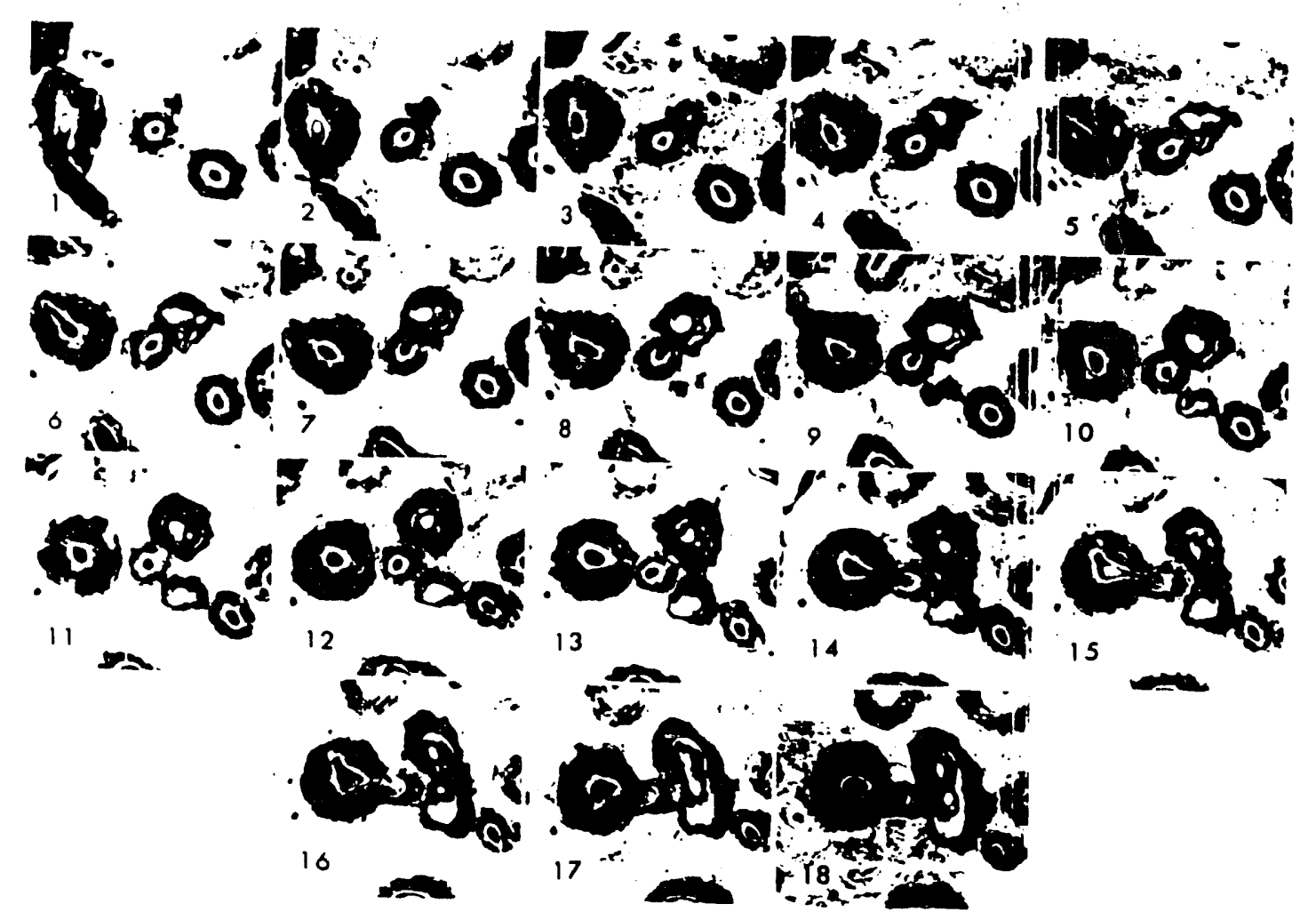

Figure 10a (frames 1-18). Photomicrographs of serial cross sections of decalcified human femur stained with silver nitrate (courtesy of Professor N.C. Tappen, Department of Anthropology, University of Wisconsin, Milwaukee). A dark resorption space is centered in the frames and gradually enlarges. The lightly stained decalcified bone is preferentially resorbed with the tunnel touching the edge of the darkly stained, less mineralized bone of the adjacent osteon but resorbing only around its peripheries.

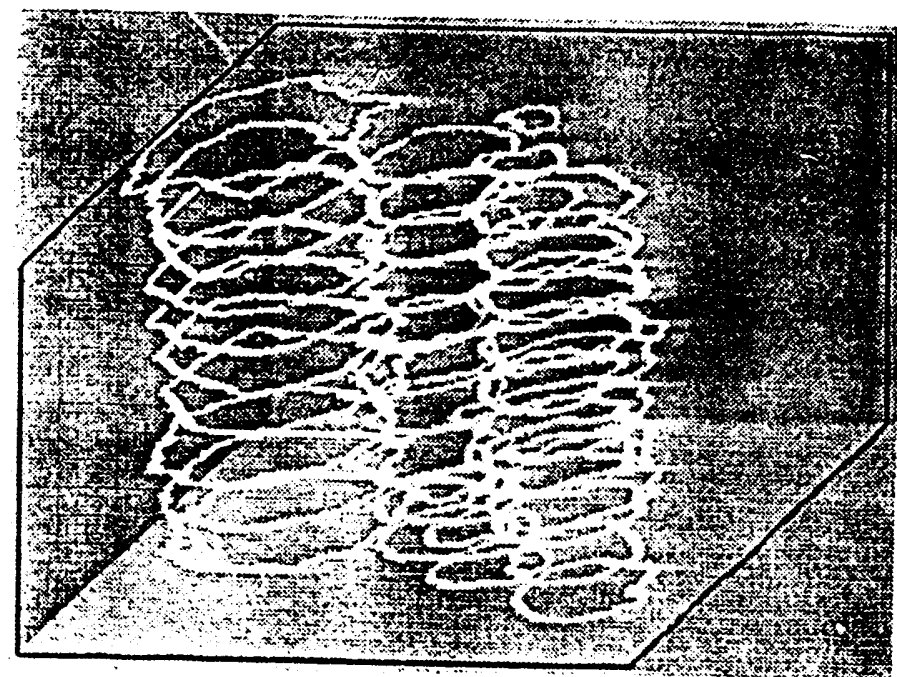

Figure $10 \mathrm{~b}$. The orientation of osteonal boundaries defined by silver nitrate staining is shown for the three mature osteons in Figure 10a which are parallel to each other and the major axis of the femur.

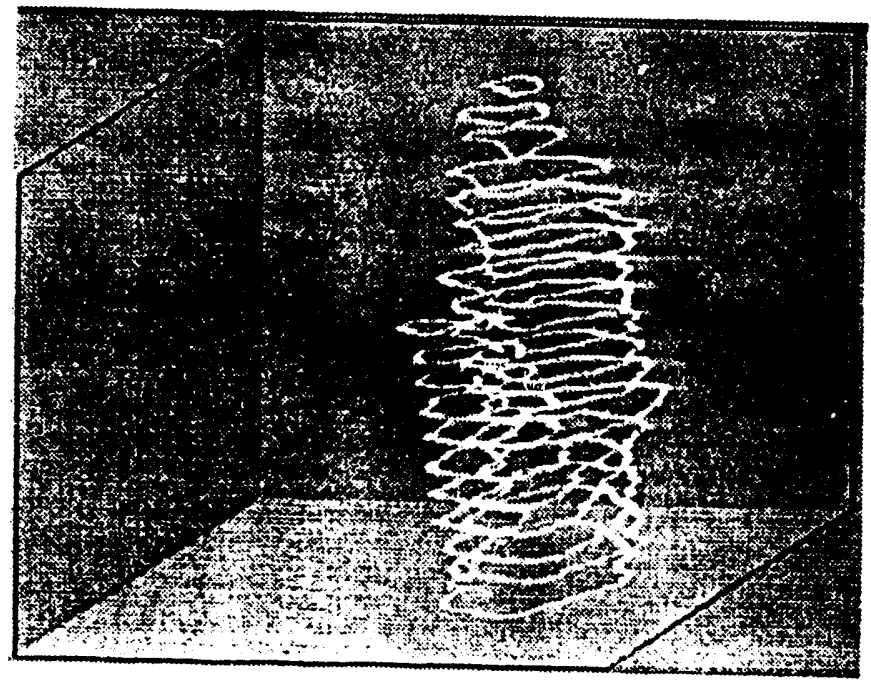

Figure 10c. The resorption tunnels that initiate in frame 1 and frame 8 ultimately coalesce in frame 13 and have a similar orientation to the mature osteons which they form around without resorbing. 


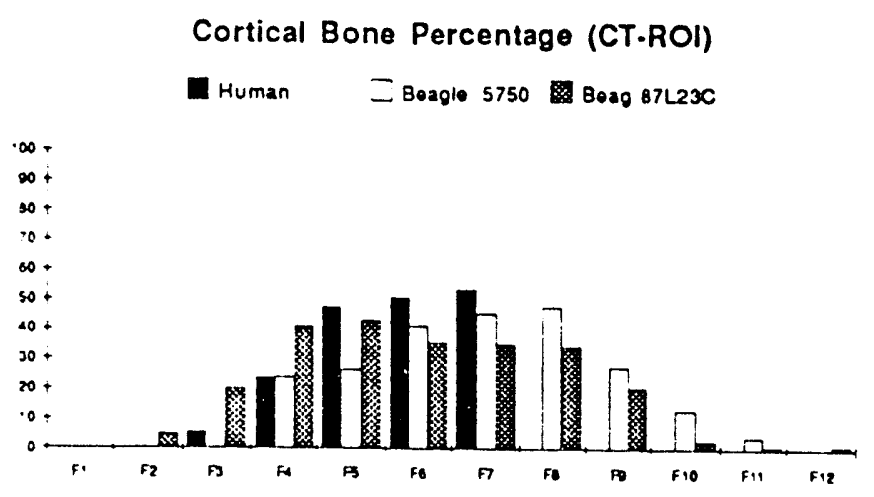

Bone Marrow Percentage (CT-ROI)

I Human ZBoagle 5750 Beag 87L230

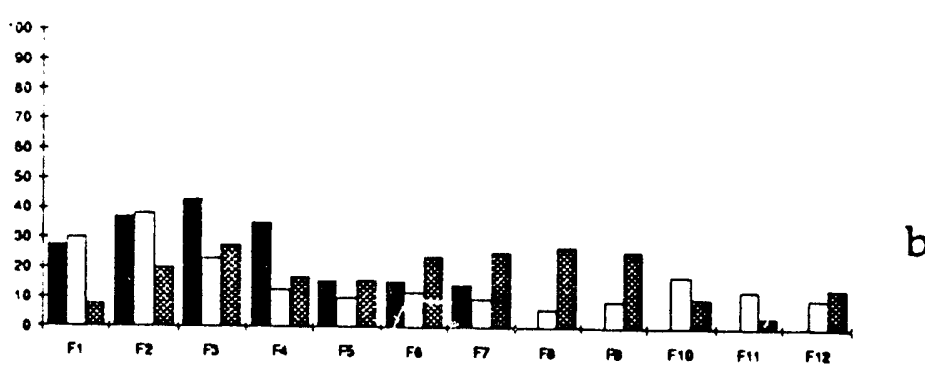

Trabecular Bone Percentage (CT-ROI)

D Human ב Beagle 5750 Boag 87L23C

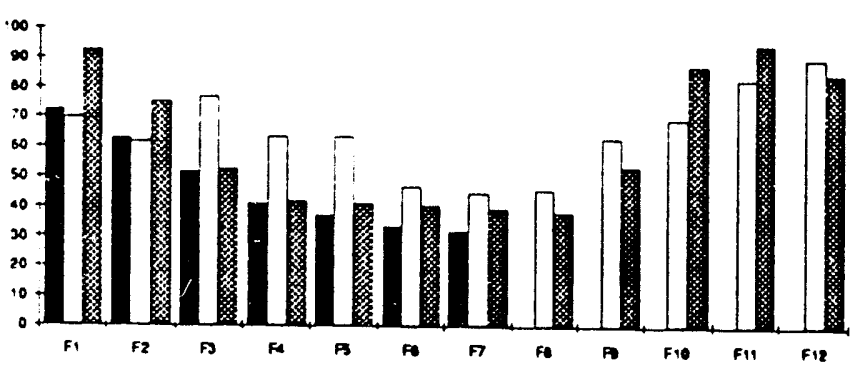

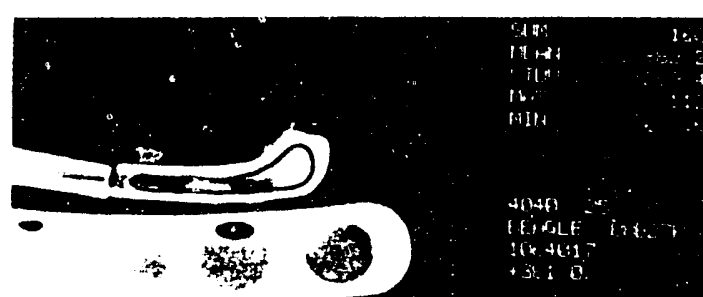

a

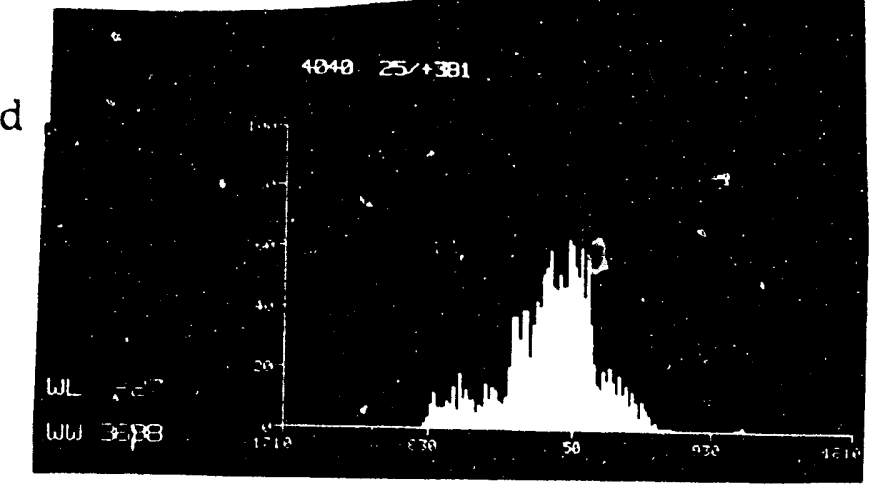

b

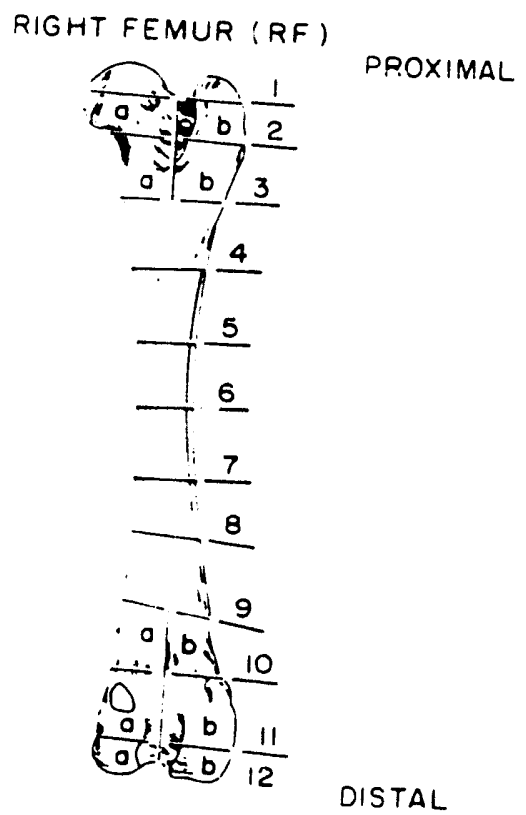

Figure 11. $(a, b, c)$ Integration over entire image regions of interest (ROI) that include all marrow, trabecular and cortical bone gives CT histograms in which the nominal CT number ROI corresponding to marrow (minus 20-150), trabecular bone (151-900. see also d), and cortical bone (901-2600) readily show the correlation of similar sections of beagle and human bone. The divisions F1 through F12 for these integrals are apportioned for both the beagle and human femur as in e. The human data is for a 78 year old male USTR case. Beagle 5750 is 125 months of age and beagle 87L23C is 15 months. The shift of some cortical bone to lower CT number nominal trabecular bone is apparent in the data of the 125 month old animal. Quantitative CT gross morphometry is done by electronic dissection of the CT images as in d, comparing cortical bone and trabecular bone ROI densities to known standards included in the CT field. 
(c) Guidance to the most radiobiologically most important skeletal regions of interest with the just completed $90 \mathrm{Sr}$ bone tumor map from life time beagle studies. Figure 12 shows the just completed skeletal map of bone tumor induced by ${ }^{90} \mathrm{Sr}$ (White et al., Joint Bone Radiobiology Workshop, Toronto July 12-13, 1991, USDOE Report UCD 472-136). The significant finding was that the tumor distribution was random with respect to simple hypothesized relationships to effective surface and bone-type mass. Earlier, Pool and associates (Pool et al., Health Physics, 44:Suppl. No.1, 155-177,1983) proposed the-as yet unchallenged, but difficult to evaluate-more complex pathways shown in Figure 13 in their paper on comparative pathogenesis of radium-induced intracortical lesions in humans and beagles. Their argument that cortical sites predominate for radiation induction of tumors by nonactinide elements (i.e., $\mathrm{Sr}$ and $\mathrm{Ra}$ ) has found consensus. The pathways start with likely decoupled aberrant bone modelling units that resorb but do not refill and lead to macroporosities (linear cortical lesions in Figure 13).

A hypothesis is that radiation is accelerating and perverting bone remodelling processes associated with normal aging-leading to both macroscopic (CT) and microscopically detectable changes with time or radiation level. We have the raw material to ferret this out. Calcified tissue with multiple fluorescent labels from young and old (18 years) beagles with and without a radioactive burden history is in our hands.

The guidance of the tumor map (Fig. 12) has focused our efforts specifically on acquiring the detailed 3-D calcified tissue fluorescent, serial stained section data, and CT (high resolution) data for the high tumor incidence regions such as the femur and humerus (proximal and midshaft) and cervical vertebrae and regions where there is radioactivity (and some remodeling), but no tumors (such as the metacarpals and low tumor incidence vertebral sites). We will install this data into an anatomical data base (Figure 14) such as that just released (September 1, 1991) for teaching (Canine Osteology by the Computer Aided Learning Facility, J. Kasper, D. Magliano, G. Cardinet, III, School of Veterinary Medicine, U.C. Davis). This particular program is based on Supercard@ (Silicon Beach) and can be expanded to include our microscopic images and tabular data just as it incorporates several levels of gross anatomy data.The information is readily recallab'e and likely all our data will fit on a few CD-ROM disks.

(d) Deposition patterns of radioactive agents that participate in apatite crystal nucleation processes in bone and leave radiation-excited electrons trapped in bone mineral. A report of this effort is in Appendix II. 


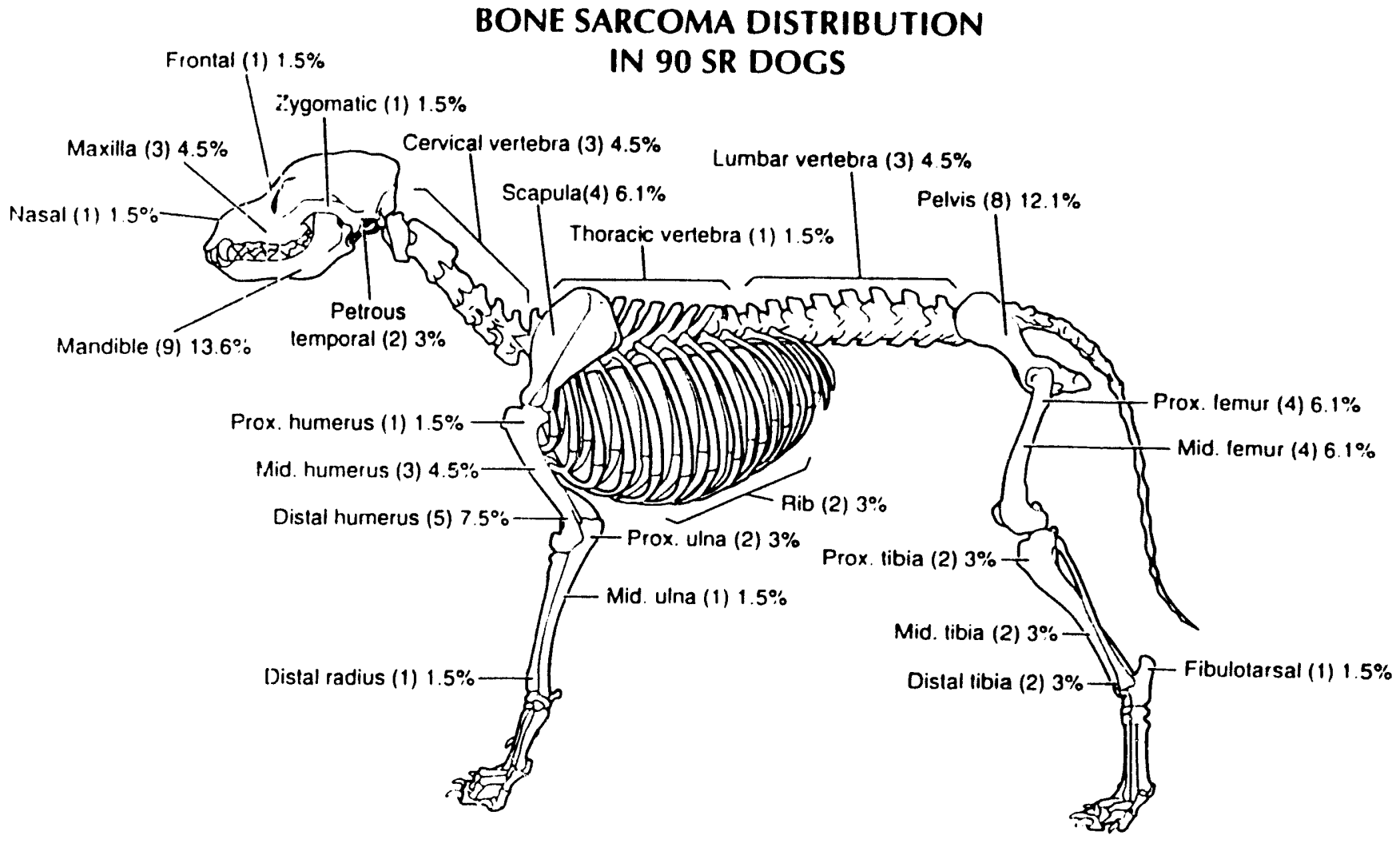

Figure 12. From White et al., Joint Bone Radiobiology Workshop, Toronto, 1991.

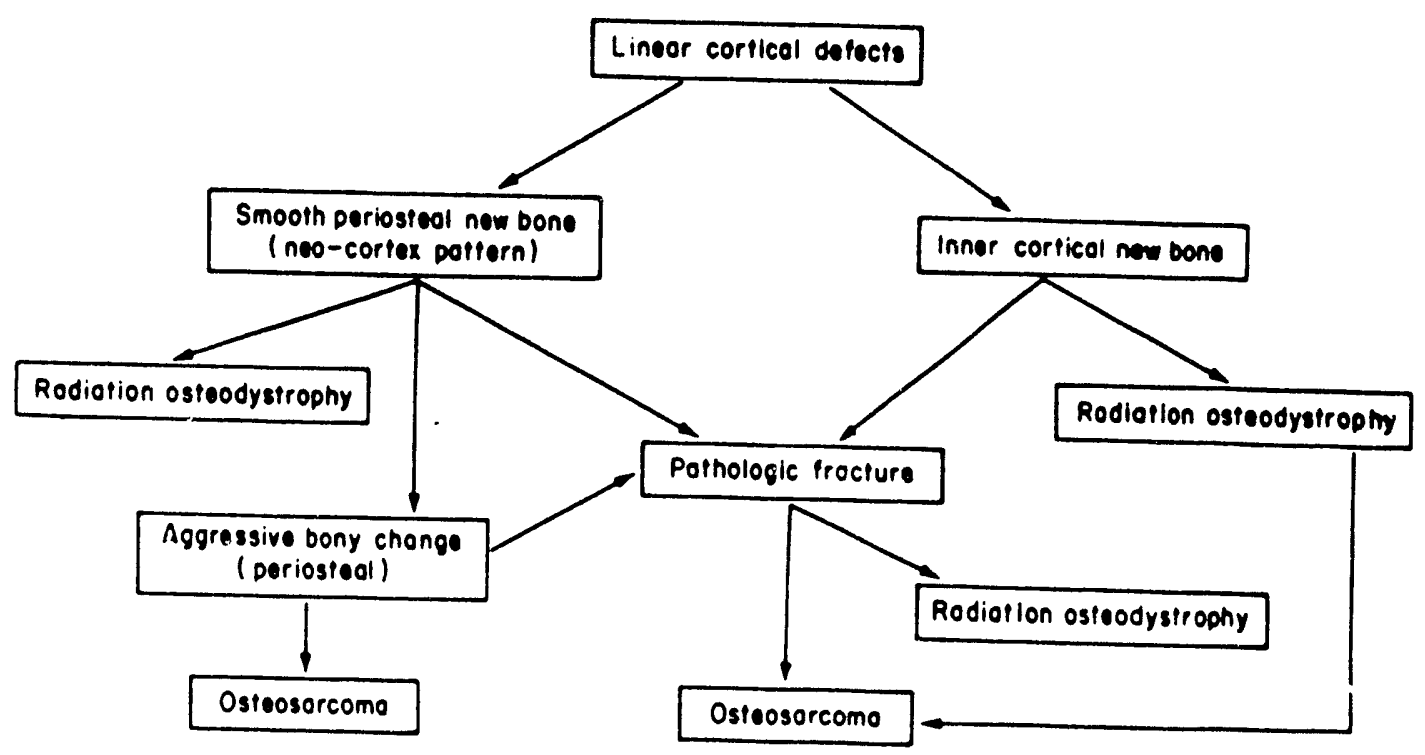

Figure 13. Schematic presentation of the sequential radiographic and histologic changes in radium-induced cortical bone lesions in beagle long bones. 

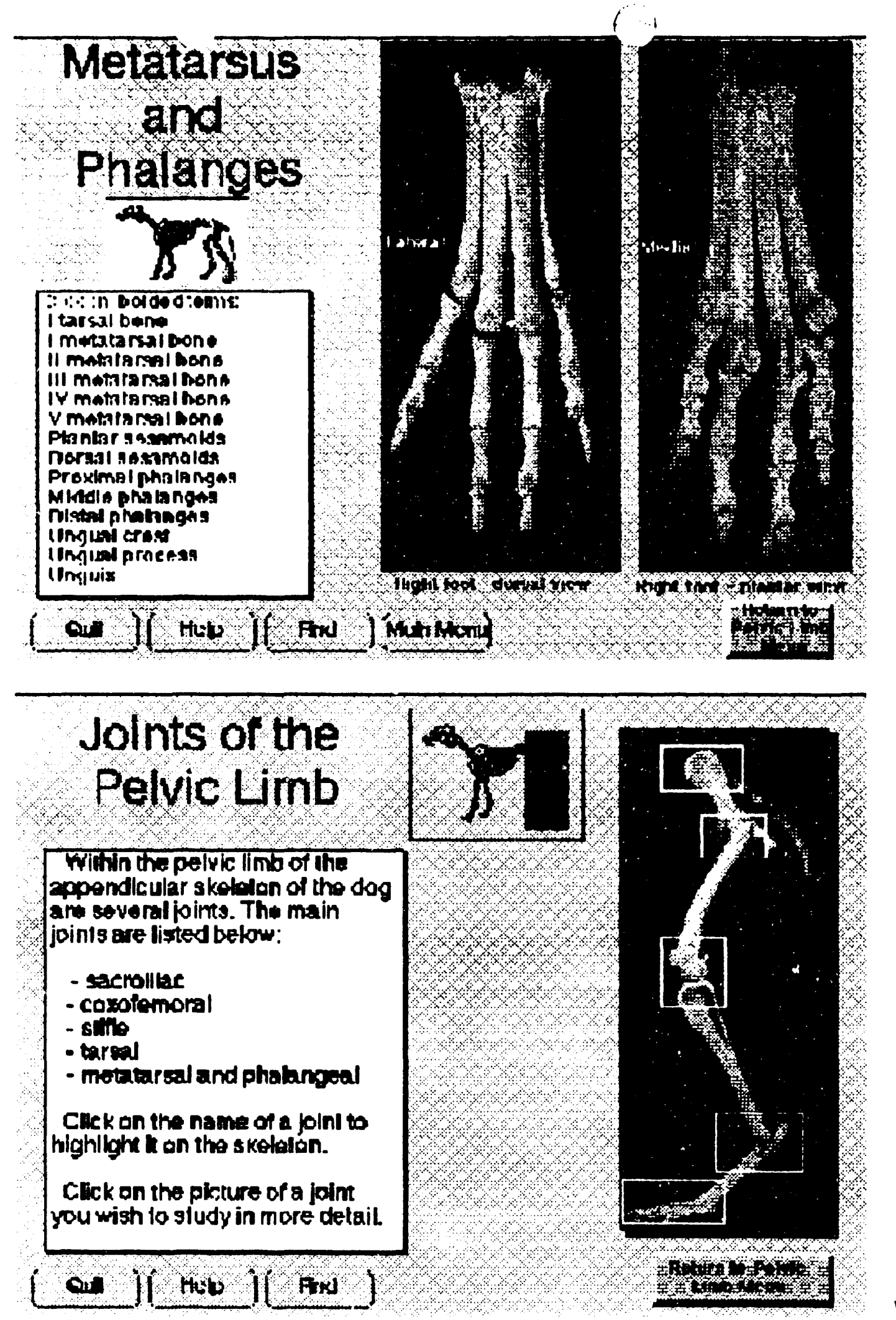

Figure 14. Representative output showing gross anatomy views of the canine skeleton taken from the Canine Osteology Program, developed by Kaspar, Magliano and Cardinet, Computer-aided Learning Facility, School of Veterinary Medicine, University of California, Davis. This program was developed with the Supercard® (Silicon Beach) database program that permits operator interaction to simply and efficiently proceed to succeedingly greater levels of detail. 


\section{Time Table}

Second six month period; Second budget period.

(1)Neutron activation analysis and dissection of young and old beagles for cross calibration with computerized tomography (CT) data. In progress.

(2)CT assay of USTR human material to survey age-related, physical activity, and disuse factors. In progress.

(3)With the tumor map experimental guide, acquire the confocal image data for fluorochrome labelled beagle bones in skeletal regions that have a high proclivity for tumor formation and those with none. Acquire and analyze the structural orientation data from physical serial sections of decalcified beagle and human bone.

(4)3-D data base modification or creation (Supercard®: Silicon Beach) that can contain image structures and tabular data.

(5)Bring the metabolic model for data on gross deposition of bone (from first budget period) seeking agents to bear on a model of total surface in cortical (and possibly trabecular bone) that is representative of the structural data from the 3D histomorphometric reconstructions.

Third budget period.

(1)Publish the design and development of confocal microscopy as a tool for bone histomorphometry.

(2)Finish the experimental data acquisition phase of the research projects in progress in the present budget period.

(3)Develop the models and present the data in a form that is useful for comparison with the multipathway, aberrant bone remodelling hypothesis put forward by Pool et al.

(4)Publish the results. 


\author{
Appendix I. Project Abstract \\ Skeletal Remodeling Dynamics: \\ New Approaches with Imaging Instrumentation
N.J. Parks, K.E. Pinkerton, J.A. Seibert, R.R. Pool
Schools of Veterinary Medicine and Medicine
University of California, Davis

The intensive investigation over the last 30 years of how, when, and where various bone-seeking radionuclides, particularly alkaline earths and actinides, produce various different anatomical and temporal distribution of bone cancer and other cellular dyscrasia has revealed gaps in our fundamental knowledge of cellular dynamics in normal bone and almost nothing about what is "normal" at late times in the lifespan. In the beagle, we are experimentally tracking the activity of cells in compact bone by microradiography and fluorescent label monitoring of remodeling and mineralization. Neutron activation analysis (NAA) and dissection are being performed with selected bones from young adult through age 18 years to determine how skeletal distribution and density of cancellous and compact bone change with age, because it is known to have a different risk potential for radiation efferts and other disease processes. In this project, we are directly comparing the information we can get from the tedious, labor intensive, and non-repeatable NAA data on a given bone to data obtained by computerized axial tomography (CAT scanning) which can, in principle, give more detailed morphological information directly in digital form, allow for repetition of the non-destructive analyses, and diminish the substantial requirement for skilled human resources. A theoretical effort to mathematically model the two-dimensional cell flux in individual osteons of young dogs is being completed and the modeling of changes with age is being compared to data from dogs fluorescently labeled at ages up to 18 years (and in one case, at 13 year intervals) so that direct assessment of remodeling with time is possible. The 3dimensional modeling of anastomosing osteon structures, the heretofore poorly characterized changes in collagen fibril orientation in filling osteons, and the density of osteocyte lacunae will be examined in young and old animals. We will specifically look for indications that radiation-induced uncoupling of osteoclastic and osteoblastic action may model aspects of spontaneously occurring osteoporosis. The Partitioned Clearance Model for radionuclide clearance in individual bones of beagles includes the bone surface and mass assumptions of the ICRP-20 model for alkaline earth metabolism in humans. The direct testing of metabolic (i.e. chemical) theory estimates of cortical and cancellous bone mass and available reactive surface for bone-seeking agents is made against the results of measurements with NAA and CAT methods for mass distribution. The combination of the morphological and metabolic models will be applied to translating the results of bone-seeking radionuclide studies in beagles to age-dependent risk estimates for human patients undergoing therapeutic procedures with bone-seeking radioactive agents. 


\section{Appendix II.}

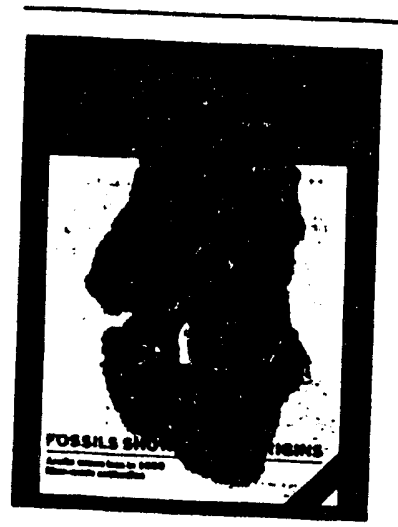

nature

24 January 1991 Vol. 349 lssue no. 6307

4 Study of two aimost complete skeletons of the Permo-Triassic Owenetta throws light on the reptile ancestry of turtles. Numerous millepede ike scavengers, found with the skel tons, may have been feeding on the two carcasses prior to burial and the ization. ization. See page 324. Photo: Diane

CONTENTS Scott.

\section{NATURE SAYS}

There are already lessons to be learnt from the Gulf War

- More GATT proposals a Inventive Britain

$287-268$

\section{NATURE REPORTS}

Saving US fusion programme $\square$ Itallan research funding increase $\square$ Lothar Späth regions $\square$ Swiss prize $\square$ License for recombinant medicine a Peer review under strain a Privatizing patents a EC failure to approve food irradiation

\section{CORRESPONDENCE}

\section{Radiation doses}

SIR-Bone-seeking radiopharmaceuticals are receiving considerable attention for therapeutic and palliative applications. including synovectomy (relief of arthritic pain) and palliative treatment of bone metastases for people with terminal cancer. Candidate radionuclides include phosphonate complexes of samarium-153, rhenium-186 and holmium-166. Clinical trials for human subjects are under way with the first two of these (ref. 1 and R. A. Holmes, personal communication).

The dosimetry for these agents relies on an accurate measurement of the activity administered. and calculations of the dose to the target organ (bone) as well as other organs. following the schema developed by the Medical Internal Radiation Dose (MIRD) committee . The critical data in this model are the physiological uptake parameters for the bone and other key organs. such as the liver and kidneys. (Such data are often available only from biodistribution studies in animals.) If the desired result is not ablation of the bone marrow. then the dose is of critical importance in limiting suppression of the immune system. The National Council on Radiation Protection and Measurements has recommended that experimental methods be developed to validate dose calculations from the MIRD model'.

We report here a new approach to experimental assessment of the radiation dose to bone using electron paramagnetic resonance (EPR) spectrometry. Ionizing radiation generates short-lived $(<1 \mathrm{\mu s})$ free radicals in soft tissues. In contrast to this. ionizing radiations which interact with mineralized bone tissue (hydroxyapatite) result in the production of a dose-dependent concentration of longlived (months to years) paramagnetic centres. The hydroxyapatite centre.

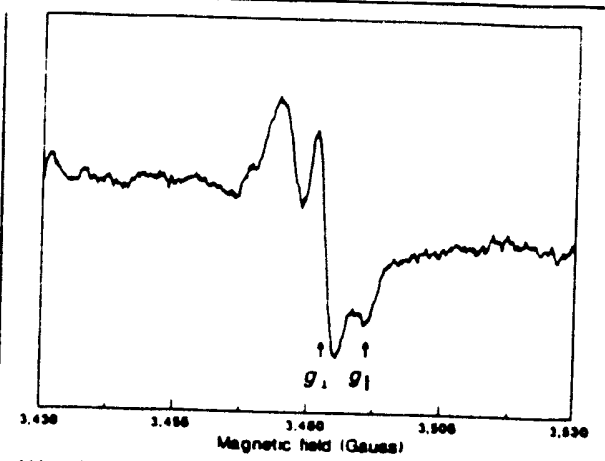

We injected a beagle with $20 \mathrm{mCl} \mathrm{kg}^{-1}$ holmium-166 $\left(t_{1 / 2}=26.8 \mathrm{~h}\right)$ as the ethylenediaminetetramethylenephosphonate complex. The animal was killed 16 days after the injection. and a humerus removed for study. Conventional dose calculations indicated a 30-Gy dose to the compact bone. We cut a fragment from the whole bone (approximate dimensions $3 \times 25 \mathrm{~mm}$ ) and put it in a $5-\mathrm{mm}$ quartz tube in an X-band microwave resonator of an EPR spectrometer. The resultant fieldswept first derivative EPR spectrum is shown. The characteristic resonances due to the hydroxyapatite paramagnetic centre are designated $a s g$. and $\mathcal{G}_{\mathbb{G}}(\boldsymbol{g}$ is the spectroscopic splitting factor). The ESR signal intensity of these resonances is a well-behaved function of absorbed dose in the bone tissue. The resonance downfield from and partially obscured by $\boldsymbol{g}$. is assigned to an organic radical. and is not considered useful for dosimetry.

detected by EPR. has been used as a marker of radiation exposure and a measure of the absorbed dose'. We have applied the EPR technique to bone tissues of an animal treated with a radiopharmaceutical to demonstrate its sensitivity towards radiation-induced centres in the mineralized tissue (see figure). We are now attempting to quantify the EPR spectral intensity in terms of absorbed dose.

Although the EPR bone dosimetry method is invasive. it does offer the first experimental technique for measuring and mapping the tissue response to the administered radiation. Given the extreme sensitivity of the bone marrow to ionizing radiation. the promise of this technique for improving bone dosimetry (for beam therapy, as well as for boneseeking radiopharmaceuticals) should be explored.

Center for Radiation Research.

M. F. DesRosiers

B. M. COURSEY

National Institute of Standards an

Technology.

Gaithersburg, Maryland 20899. USA

\author{
M. J. AvILA \\ N. J. PARKS \\ Laboratory for Energy-Related Health \\ Research. \\ University of California, Davis. \\ California 95616. USA
}

1. Maxon. H.R. ef al. Radiology 178. 155-159 (1990)

2. Loevinger. R.. Budinger. T.F \& Watson. E.E. MIRD Primer for Absorbed Dose Calculations isociety of Nuclear Medt Cine, New York, 1988)

3. NCRP Report 83 (NCRP. Bethesaa. Maryland. 1985).
4 Desrosiers. M.F. Nature 348. 485 (1990). 

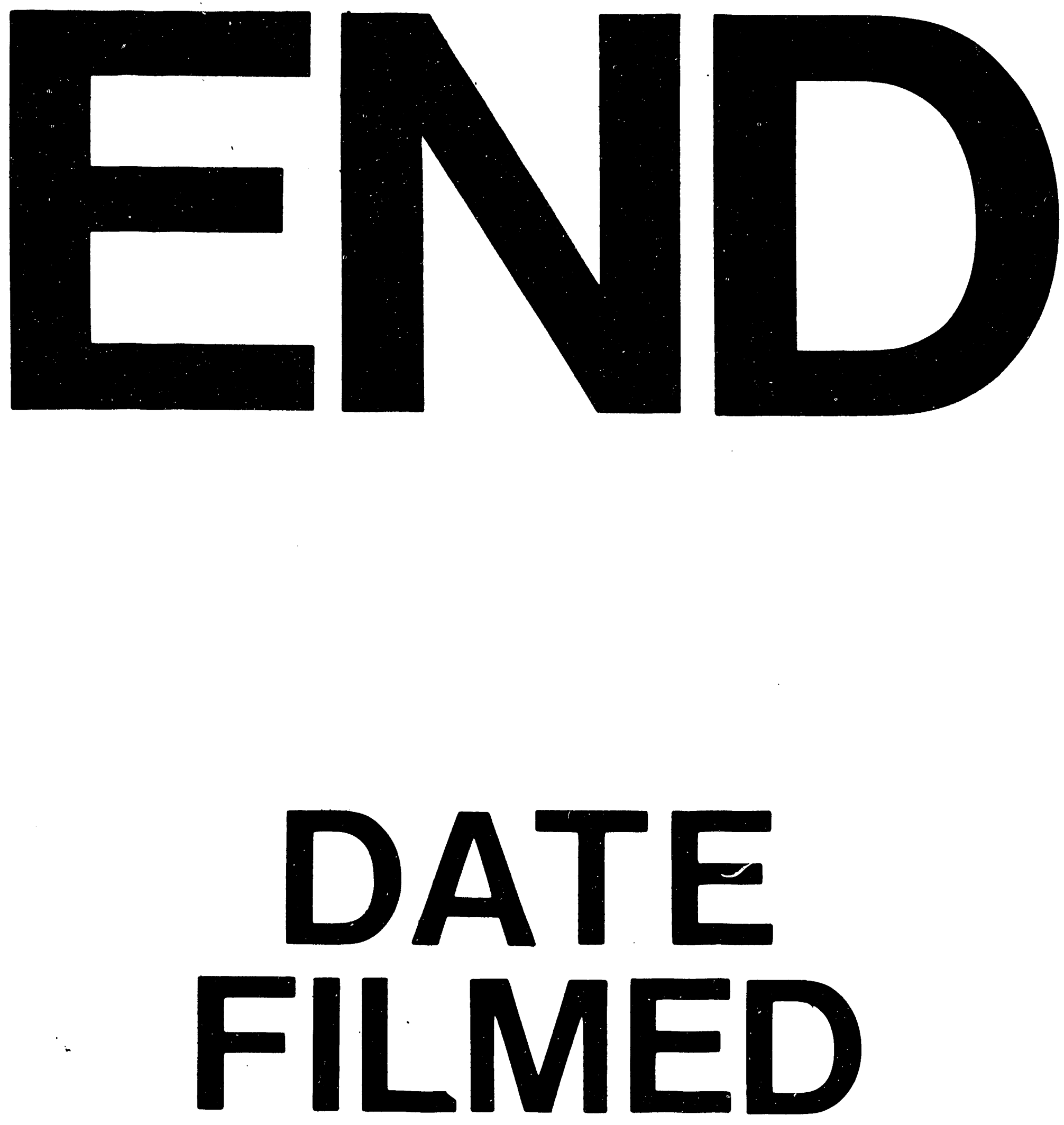

$t$

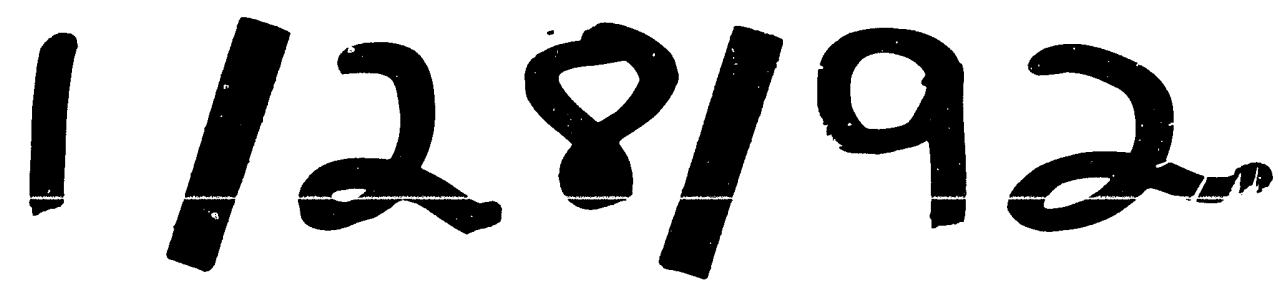




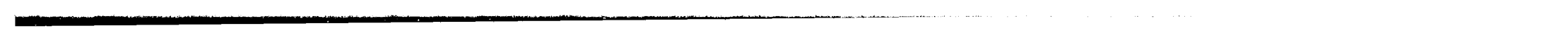

